\title{
A Qualitative method to determine the loss of efficiency in the DELPHI Barrel RICH MWPC's due to ageing
}

\author{
G. van Apeldoorn ${ }^{1}$, C. Bourdarios ${ }^{2}$, M. Davenport ${ }^{3}$, M. Dracos ${ }^{4}$, \\ P. Juillot ${ }^{4}$, N. Kjaer ${ }^{3}$, D.Z. Toet ${ }^{1}$, A. Tsirou ${ }^{3}$
}

\begin{abstract}
We describe a simple method to survey effects of ageing in the Multiwire Proportional Chambers of the DELPHI Barrel Ring Imaging CHerenkov (RICH) counters. These chambers detect single photoelectrons created by Cherenkov light a photosensitive (TMAE loaded) gas mixture. They receive as well the much larger amount of ionisation electrons $-\sim 60 / \mathrm{cm}$ - created by charged particles. The method essentially consists of a comparison of the plateau curves measured after each year's running period in order to detect changes in their characteristics as a function of exposure.
\end{abstract}

Presented at the 3rd London Conference on Position Sensitive Detectors, London, 6-10 September 1993.

Submitted to Nuclear Instruments and Methods in Physics Research.

\footnotetext{
${ }^{1}$ NIKHEF-H, Postbus 41882, NL-1009 DB Amsterdam

${ }^{2}$ LAL, Laboratoire de l'Accélérateur Linéaire, Université de Paris-Sud (Paris XI), Bâtiment 200, F-91405 Orsay Cedex

${ }^{4}$ CRN, Centre de Recherches Nucléaires, IN2P3-CNRS/ULP, B.P. 20, F-67037 Strasbourg Cedex
} 

The DELPHI experiment [1, 2] at LEP incorporates Ring Imaging Cherenkov (RICH) counters in order to allow charged hadron identification $[1,3,4]$. The system is composed of a detector in the central region of the experimental setup, referred to as the Barrel RICH, and of detectors in the forward-backward regions, the Forward RICH. In the Barrel RICH, Cherenkov light is intercepted by quartz tubes filled with a photosensitive gas. The photoelectrons created there drift in an electric field towards an array of Multiwire Proportional Chambers (MWPC's), one at the end of each drift tube [5]. In this paper we describe a method to monitor possible ageing of the wires of these chambers.

Ageing has been the subject of many investigations [6]. Some of these go beyond the scope of a particular design, studying ageing as a function of gas composition, gas flowrate, temperature, wire diameter and wire material, intensity, etc.. Ageing depends on the accumulated charge on the electrodes relevant for the so-called gas amplification process (e.g. anode wires) and is thus conveniently studied as a function of the total accumulated charge per unit (wire-) length. It is manifest in a shift to lower values of the pulse height distribution or in the decrease of anode current. Upon inspection aged wires are generally found to be coated by deposits of chemical products, which upon analysis generally show a strong presence of $\mathrm{C}, \mathrm{N}$ and $\mathrm{O}[6]$. These products are created in the avalanches from the different molecules present in the chamber gas, including of course possible impurities and are responsible for the loss in gain, i.e. for the decrease of the number of electrons created in the avalanches.

\section{The Barrel RICH MWPC's and the Drift Gas}

The Barrel RICH MWPC's have been extensively described elsewhere [5]. We repeat here the main characteristics and indicate the materials used in their construction (fig.1):

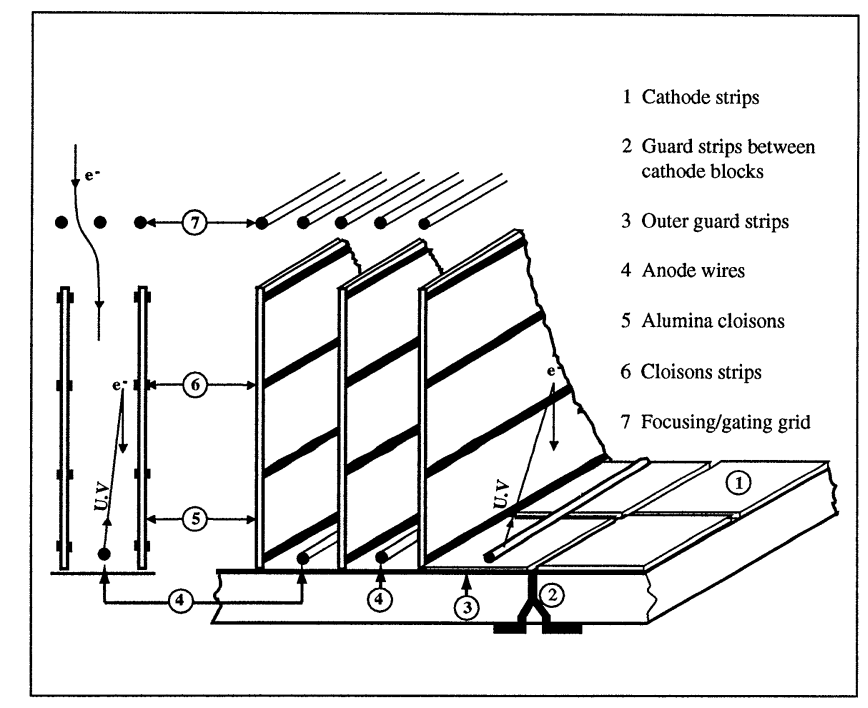

Figure 1: Schematic layout of the Barrel RICH MWPC's

- The chambers are designed to detect single photoelectrons. To that order they have a gain factor of $2.10^{5}$ at the working point, i.e. at a cathode voltage of $-1450 \mathrm{~V}$ [7].

- The anode wires are $20 \mu \mathrm{m}$ diameter gold-plated tungsten wires, their pitch is $2.62 \mathrm{~mm}$ and they are positioned at $0.5 \mathrm{~mm}$ from the flat cathode plane. 
- The cathode plane is subdivided into $3.8 \mathrm{~mm}$ wide/4 $\mathrm{mm}$ pitch gold plated copper strips on a G10 printed circuit board.

- Blinds are placed between the wires as an optical shielding against secondary (feedback) photons created in the avalanches. They consist of $9.8 \mathrm{~mm}$ high, $0.254 \mathrm{~mm}$ thick alumina sheets and are equipped with potential strips on each side to focus the drifting electrons onto the anodes.

The chamber gas is a mixture of methane and ethane in the ratio $3: 1$; by bubbling this mixture through a bubbler filled with TMAE (Tetrakis diMethyl Amine Ethylene) [8] at a fixed temperature (normally $28^{\circ} \mathrm{C}$ ), it is doped with photo-sensitive TMAE vapour at the saturation pressure of 0.8 mbar.

\section{Description of the Method}

The well known and proven method to obtain a quantative measurement of the effect of ageing is of course the measurement of the shift of the pulse height distribution as a function of the accumulated charge. Such measurements have been performed on a prototype chamber in a test beam [9]. Our conclusion was that the gain ("gas amplification") would decrease to $50 \%$ of that of a new chamber not before having accumulated $2.510^{-4} \mathrm{C} / \mathrm{cm}$.

At present our (digital, FASTBUS-based) readout system is not equipped with ADC's. With these we would have been able to monitor for some of our 48 MWPC's the pulseheight distributions using e.g. the calibration system [4]. Instead we have chosen a solution which consists in measuring the plateau curves (representing the number of hits as a function of voltage) after each year's running period. Ageing will show in a change of the characteristics of this plateau curve, notably in a shift of the voltage at which the chamber starts to count and that where the plateau starts.

For these measurements we maintained the same conditions as during normal running (gas composition, TMAE temperature, flowrate and pressure). The temperature in the Barrel RICH was $40^{\circ} \mathrm{C}$, variations of more than $0.2^{\circ} \mathrm{C}$ were seldom. The pressure in the drift tubes was kept at $1038 \mathrm{mb}$. The calibration system [4] was used to produce single photoelectrons in the drift tubes; the light conducted by the each of the $45 \mathrm{UV}$-fibres equipping each of the drift tubes gives rise to the creation of an average of 0.1 photoelectron per pulse of an external UV lamp. The plateau curve was measured varying the cathode voltage between $-1100 \mathrm{~V}$ and $-1500 \mathrm{~V}$ with steps of $25 \mathrm{~V}$. Voltages of the blinds, the gating wires, the transfer electrode and the drift field were varied accordingly. At each of these voltages we simply record the number of wire hits in each of the chambers.

In our study we compare the behaviour of chambers after different ageing (running-) periods. Since the chambers on one side of the Barrel RICH were operational one runningyear earlier than the chambers on the opposite side, we can compare the ageing effects of these chambers. The two sides are called (for topological reasons) side A and side $\mathrm{C}$ respectively -see table 1 and figure 2. At each side of the Barrel RICH two chambers are kept at constant (working point) voltages. These chambers are used to monitor any variations with time of the light yield from the two external lamps (one lamp for each side).

Since the calibration system does not distribute the same amount of light to each drift tube, counting rates at a given voltage differ between the chambers. We therefore normalize the counting rate of all chambers to give the same value at $-1450 \mathrm{~V}$.

The chambers used for the study are chambers that did not undergo an intervention (remained in situ) in the periods considered. 
The plateau curves measured after the different running periods are shown in fig. 2 . The results of straight line fits to the rise of the curve and to the plateau part of it are given in table 1 .

\begin{tabular}{||c|c|c|c||}
\hline \hline chambers,age & $\begin{array}{c}\text { gain starting point } \\
(\mathrm{V})\end{array}$ & $\begin{array}{c}\text { slope of rise } \\
\text { (hits/V) }\end{array}$ & $\begin{array}{c}\text { intersect. with } \\
\text { plateau line }\end{array}$ \\
\hline \hline $\begin{array}{c}\text { side A '92 } \\
\text { '3 years' } \\
\text { side C '92 } \\
\text { '2 years' } \\
\text { side A '91 } \\
\text { '2 years' } \\
\text { side C '91 } \\
\text { '1 year' }\end{array}$ & $1178 \pm 47$ & $4.83 \pm 0.13$ & $1276 \pm 66$ \\
\hline \hline
\end{tabular}

Table 1: Characteristics of plateau curves measured after different periods of ageing. Column 2 gives the intersection of the line fitted to the 'rise' with the 'zero hits' line. Column 3 gives the intersection of the line fitted to the 'rise' with the line fitted to the plateau.

No ageing effects are visible. The curves show that there is no shift to higher voltages of the point where the chambers start to count. This is confirmed by what is shown in the electron-microscope photographs (fig.3), one of a (3 run-years) old central wire which has received $>10^{7}$ electrons $/ \mathrm{cm}$ and one of a new wire of the same material. The results of the X-ray spectrographic analyses are also virtually identical.

\section{Conclusions}

We have described a simple method to monitor the effects of ageing in our MWPC's on the counting efficiency. It is a method of which the result is directly repesentative and applicable to our readout method in view of the fact that we use counting and do not read out pulseheights in our detector. The results we measured are conform our expectations and we will certainly continue to exploit this method in the future, where ageing effects might become more pronounced.

\section{References}

[1] DELPHI Technical Proposal, CERN/LEP 83-3 (1983).

[2] P.Aarnio et al., Nucl. Instr. and Meth. A303 (1991) 233.

[3] Barrel RICH Construction Group, DELPHI 86-22 RICH-22 (1986).

E.G.Anassontzis et al., IEEE Trans. Nucl. Sci. NS-38 (1990) 417.

[4] E.G.Anassontzis et al., Nucl. Instr. and Meth. A323 (1992) 351.

[5] D.Bloch et al., Nucl. Inst. and Meth. A273 (1988) 847.

G. van Apeldoorn et al., Nucl. Inst. and Meth. A283 (1989) 767.

[6] J.Va'vra, NIM A252(1986)249.

J.Va'vra, SLAC-PUB-4116 (1986) and Paper presented at the 1986 Nucl. Science Symp. Washington D.C.

J.Va'vra, CRID MEMO nr.36 (1987). 
R.Henderson et al.TRI-PP-87-84 and Paper presented at the 1987 Nucl. Science Symp. S.Francisco.

K.Martens et al., Paper presented at the First Workshop on RICH Detectors, Bari (1993).

J.Kadyk, Nucl. Inst. and Meth. A300 (1991) 436.

[7] M.Dracos and D.Loukas, Nucl. Inst. and Meth. A302 (1991). 241.

[8] R.A.Holroyd et al., Nucl. Inst. and Meth. A261 (1987) 440.

[9] D.Langerveld, D.Toet, W.Dulinski, Aging due to TMAE in a MWPC, Internal Report (unpublished) (1987). 


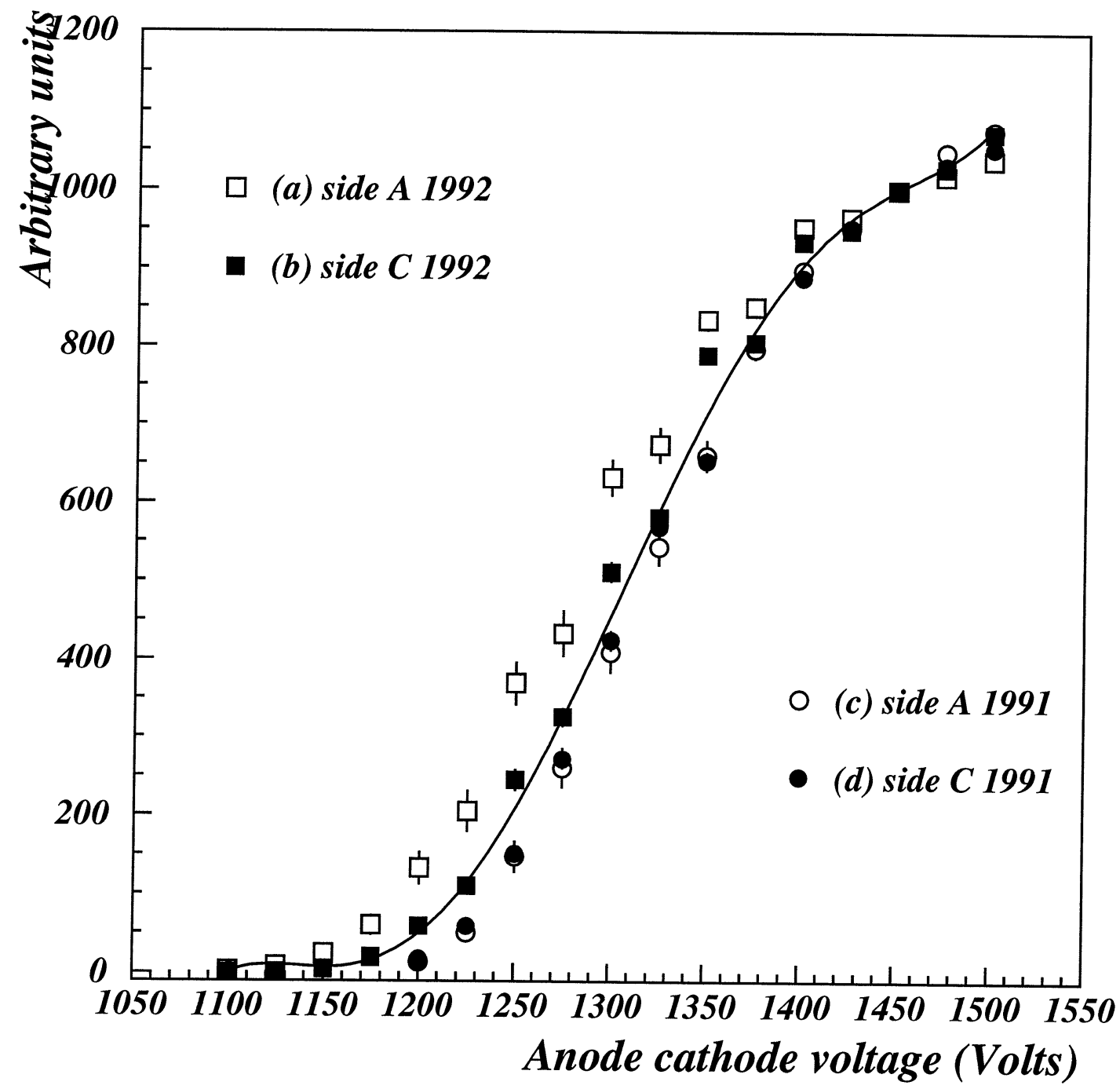

Figure 2: Plateau curves for side A end 1992, i.e after 3 'runyears' (a); side C 1992 (2 runyears) (b); side A 1991 (2 runyears) (c); side C 1991 (1 runyear) (d). 


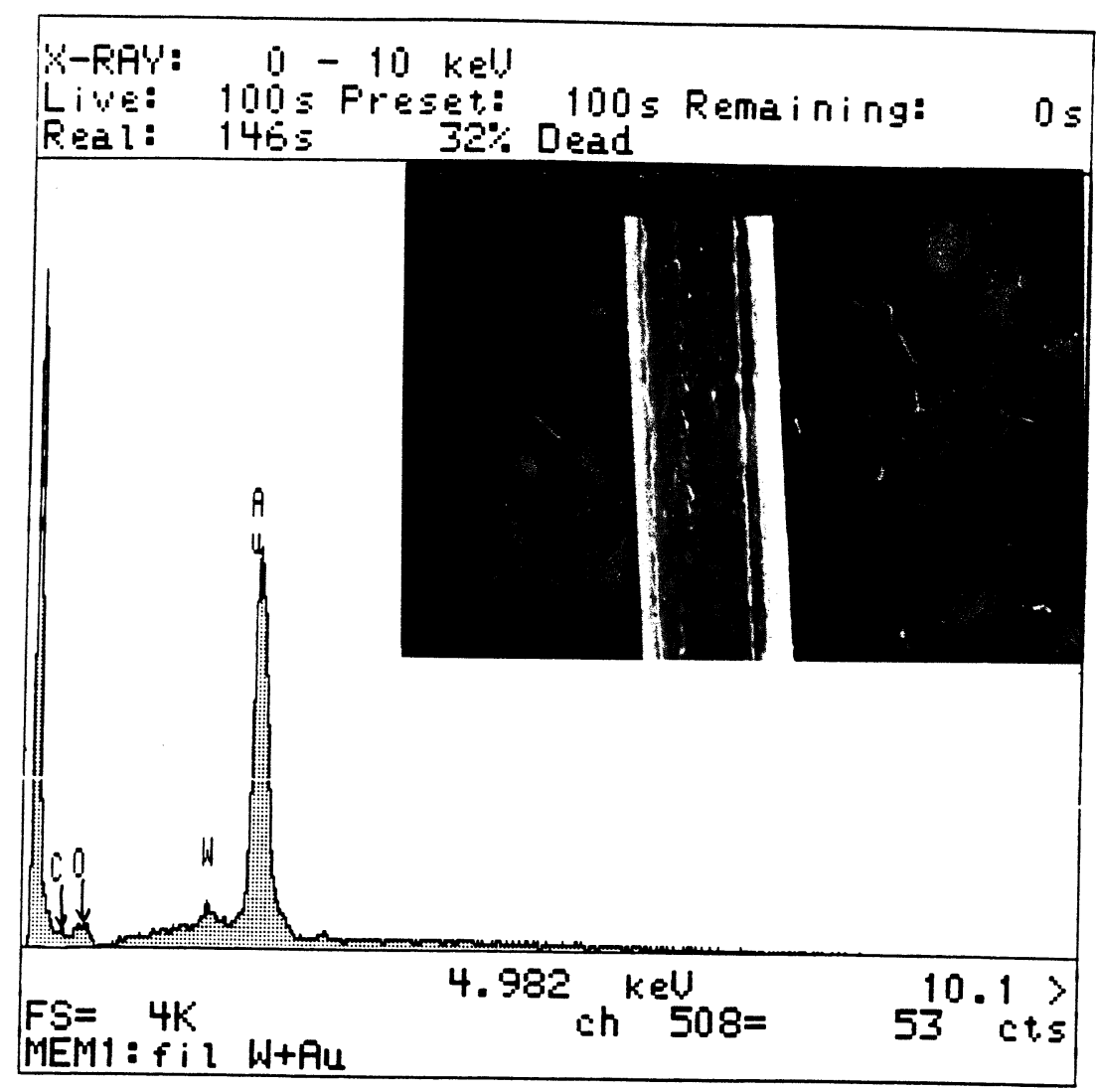

a) central anode wire after 3 running periods

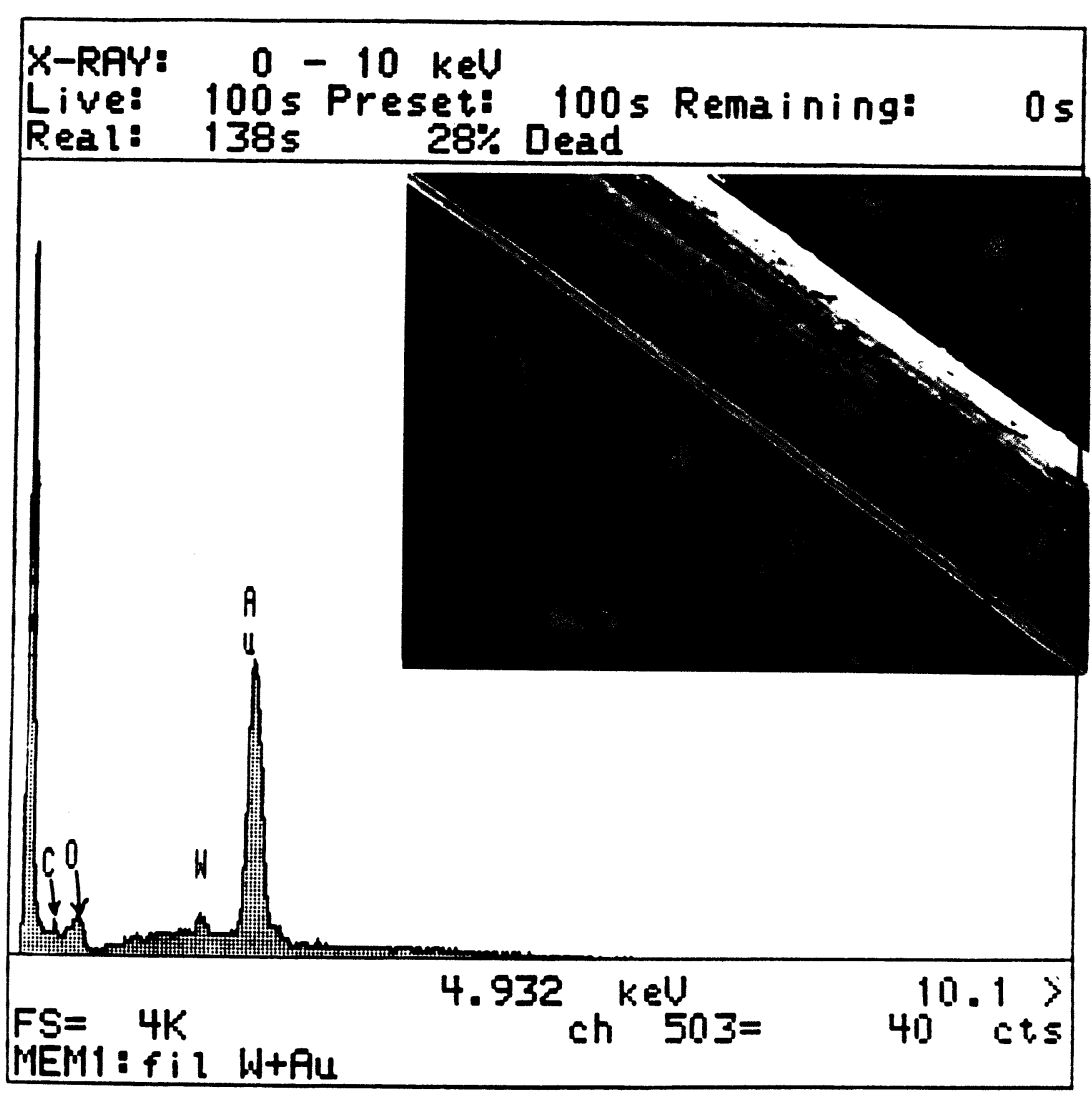

b) unused wire (for comparison)

Figure 3: Scanning electron microscope photographs and X-ray spectra of a wire aged for 3 running periods (a) and of a new wire (b). 